\title{
Effect of nutrient deficiencies on in vitro Th1 and Th2 cytokine response of peripheral blood mononuclear cells to Plasmodium falciparum infection
}

Erasto V Mbugi ${ }^{1,7^{*}}$, Marjolein Meijerink ${ }^{1,2}$, Jacobien Veenemans ${ }^{1}$, Prescilla V Jeurink ${ }^{1,3}$, Matthew McCall ${ }^{4}$, Raimos M Olomi ${ }^{5}$, John F Shao ${ }^{5}$, Jaffu O Chilongola ${ }^{5}$, Hans Verhoef ${ }^{1,6}$, Huub FJ Savelkoul $^{1}$

From Parasite to Prevention: Advances in the understanding of malaria

Edinburgh, UK. 20-22 October 2010

\section{Background}

An appropriate balance between pro-inflammatory and anti-inflammatory cytokines that mediate innate and adaptive immune responses is required for effective protection against human malaria and to avoid immunopathology. In malaria endemic countries, this immunological balance may be influenced by micronutrient deficiencies. Peripheral blood mononuclear cells from Tanzanian preschool children were stimulated in vitro with Plasmodium falciparum-parasitized red blood cells to determine $\mathrm{T}$-cell responses to malaria under different conditions of nutrient deficiencies and malaria status. The data obtained indicate that zinc deficiency is associated with an increase in TNF response by $37 \%$; $95 \%$ CI: $14 \%$ to $118 \%$ and IFN- $\gamma$ response by $74 \%$; $95 \%$ CI: $24 \%$ to $297 \%$. Magnesium deficiency, on the other hand, was associated with an increase in production of IL-13 by $80 \%$; $95 \%$ CI: $31 \%$ to $371 \%$ and a reduction in IFN- $\gamma$ production. These results reflect a shift in cytokine profile to a more type I cytokine profile and cellcell mediated responses in zinc deficiency and a type II response in magnesium deficiency. The data also reveal a non-specific decrease in cytokine production in children due to iron deficiency anaemia that is largely associated with malaria infection status. Figure 1 and Figure 2.

\section{Conclusions}

The pathological sequels of malaria potentially depend more on the balance between type I and type II cytokine responses than on absolute suppression of these cytokines and this balance may be influenced by a combination of micronutrient deficiencies and malaria status.

\section{Author details}

${ }^{1}$ Cell Biology and Immunology Group, Wageningen University, The Netherlands. ${ }^{2}$ Host-Microbe Interactomics,Wageningen University, The Netherlands. ${ }^{3}$ Danone Research, Wageningen, The Netherlands. ${ }^{4}$ Department of Medical Microbiology, Radboud University, Nijmegen, The Netherlands. ${ }^{5}$ Kilimanjaro Christian Medical Centre (KCMC), Moshi, Tanzania. ${ }^{6}$ London School of Hygiene and Tropical Medicine, Nutrition and Public Health Intervention Research Unit, London, UK. ${ }^{7}$ Muhimbili University of Health and Allied Sciences, Biochemistry Department, School of Medicine, Dar es Salaam, Tanzania.

\section{Published: 20 October 2010}

\section{References}

1. Aultman KS, Gottlieb M, Giovanni MY, Fauci AS: Editorial:

Anophelesgambiae genome: completing the malaria triad. Science 2002, 298:13.

2. Riley EM, Wahl S, Perkins DJ, Schofield L: Regulating immunity to malaria. Parasite Immunol 2006, 28:35-49.

3. Perlmann $P$, Troye-Blomberg M: Malaria and the immune system in humans. Chem Immunol 2002, 80:229-242.

4. Riley EM: Is T-cell priming required for initiation of pathology in malaria infections? Immunol Today 1999, 20:228-233.

5. Stevenson MM, Riley EM: Innate immunity to malaria. at Rev Immunol 2004, 4:169-180.

doi:10.1186/1475-2875-9-S2-P21

Cite this article as: Mbugi et al.: Effect of nutrient deficiencies on in vitro Th1 and Th2 cytokine response of peripheral blood mononuclear cells to Plasmodium falciparum infection. Malaria Journal 2010 9(Suppl 2):P21. 

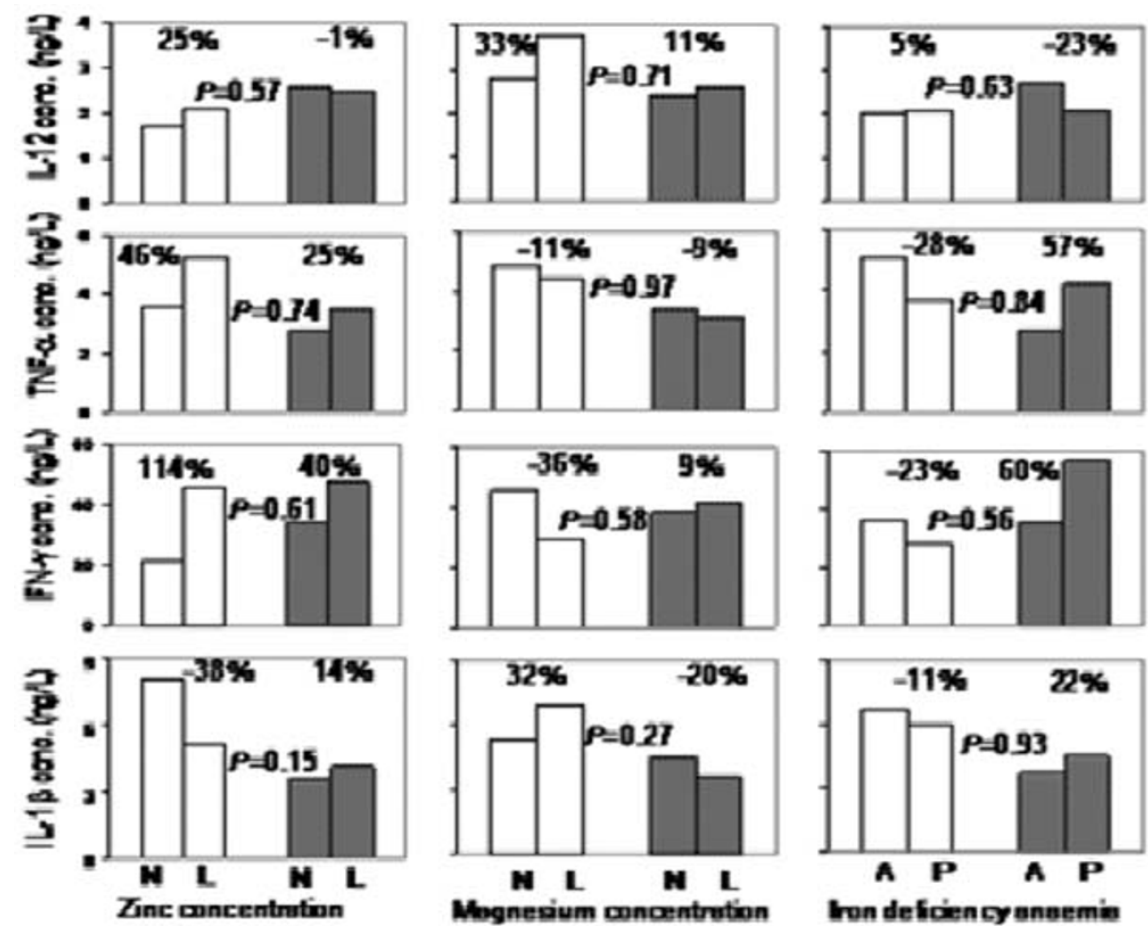

Figure 1 Associations between micronutrient status and supernatant type I cytokine concentrations following 7 days of PEMCS stimulation with Plasmodium falciparum-infected erythrocytes by malaria infection status of the child at the time of blood collection. N: nutrient concentrations; L; low concentrations; A: absent; P: present. Percentage indicates paired group difference in cytokine concentrations. Data from children without and with malaria infection at the time of blood collection are indicated with open and shaded bars respectively.
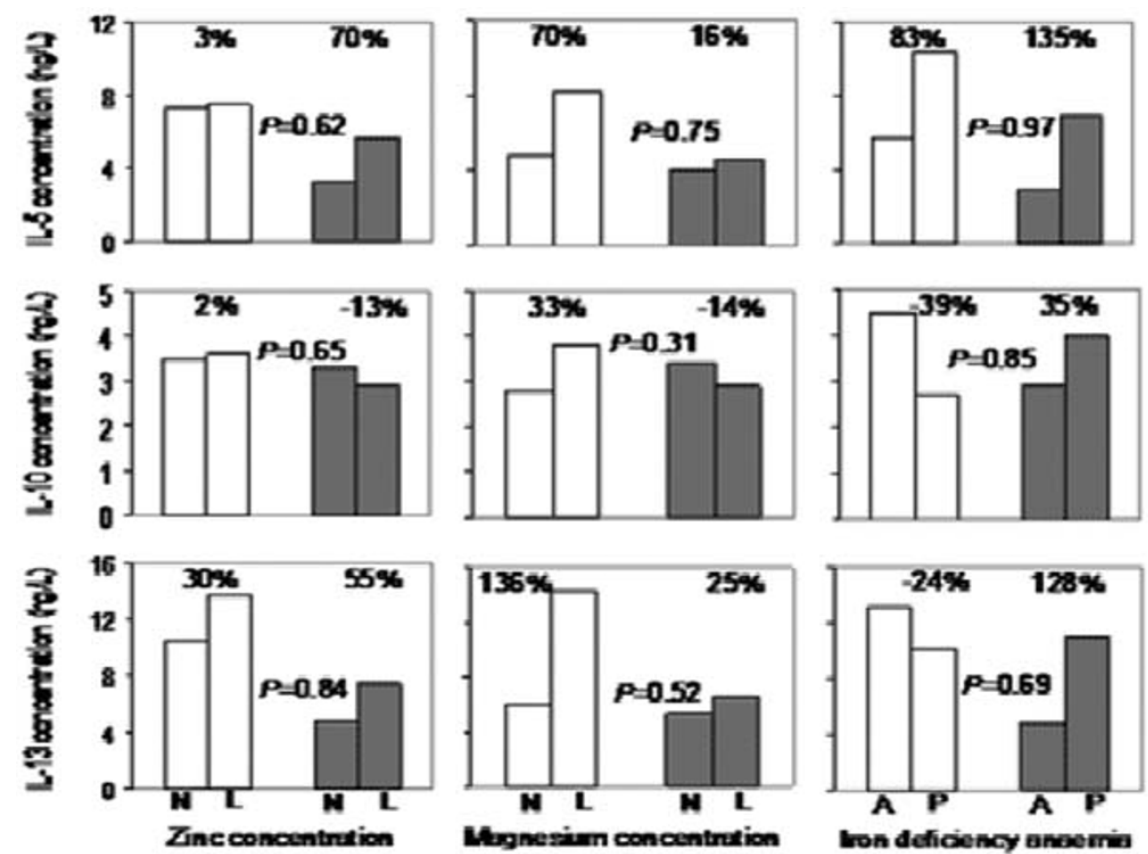

Figure 2 Associations between micronutrient statues and supernatant type II cytokine concentrations following 7 days of PEMCs stimulation with Plasmodium falciparum-infected erythrocytes by malaria infection status of the child at the time of blood collection. N: nutrient concentrations; L; low concentrations; A: absent; P: present. Percentage indicates paired group difference in cytokine concentrations. Data from children without and with malaria infection at the time of blood collection are indicated with open and shaded bars respectively. 